in Austria, which means a total market share of $33 \%$ of all bikes sold (2016: 22\%).

E-bikes are above all a good alternative for motor vehicles in city traffic.

The KFV (Austrian Road Safety Board) is currently dealing with questions about the safety of e-bikes.

Methods In our studies the following methods were applied:

1. analysis of existing accident data on e-bikes (statistical data, media analysis)

2. on-site observations (e.g. helmet wearing quota for cyclists, collection of speed differences of selected bike types, ...)

3. questionnaire survey among 101 e-bike users concerning the comfort and safety of different bike types

4. survey on the subjective safety of e-bike users and e-bike non-users

Results Results showed among others that...

1. ... in 20181.025 injured and 17 killed e-bike cyclists showed up in statistics

2. ... helmet wearing rate of e-bike cyclists was $44 \%$ (compared to $25 \%$ of conventional bikes)

3. ... the average speed of pedelecs was $21.4 \mathrm{~km} / \mathrm{h}$ (compared to $18 \mathrm{~km} / \mathrm{h}$ of conventional bikes)

4. ... pedelec drivers feel safer and more comfortable than conventional cyclists

Conclusion Based on the results, tips for safe driving with ebikes were drawn up and measures and recommendations for action to increase the safety of e-bike users were derived.

\section{P5.009 PREDICTORS OF PSYCHOLOGICAL AND FUNCTIONAL OUTCOME FOLLOWING TRAFFIC INJURIES: A SCOPING REVIEW}

${ }^{1}$ Rayan Alharbi*, ${ }^{1}$ Charne Miller, ${ }^{2}$ Virginia Lewis. 'La Trobe Alfred Health Clinical School, La Trobe University, Prahran, Australia; ${ }^{2}$ Australian Institute for Primary Care and Ageing, La Trobe University, Bundoora, Australia

10.1136/injuryprev-2021-safety.229

Background Each year tens of millions of individuals are injured or disabled as a result of Road Traffic Crashes (RTCs). In addition to immediate death, there is an increased risk of subsequent death, ongoing physical disability, psychological issues, and reduced overall health related quality of life following RTCs. This study aims to identify the factors reported in the literature that are associated with adult trauma patients' morbidity following an RTC.

Methods A scoping literature review was conducted. Peerreviewed articles were retrieved from MEDLINE/PubMed, EMBASE, and CINAHL.

Results This literature review identified six categories of variables being used in studies that explored predictors and factors associated with physical and psychological morbidity following RTCs. Five of the categories represented independent variables; (i.) injury characteristics and hospital predictive factors; (ii.) demographic factors; (iii.) family and social support; (iv.); compensation system process and fault in the RTC (v.); and pre-injury health status (vi.). The sixth category was used to represent the range of (vi.) psychological and functional outcomes.

Conclusion These findings highlight the multiple and diverse contributors that influence person outcomes following an
RTC. These factors are intrinsic and extrinsic and commence from the time of injury as well as highlighting the importance for ongoing support after acute care discharge to enable a quick return to optimal wellbeing.

Learning Outcomes Research examining RTC outcomes must integrate information about the crash response and health care system whilst simultaneously measuring other factors to appropriately quantify the relative contribution of each variable to psychological and functional outcomes.

\section{P5.010 RED LIGHT RUNNING RATE IN THE KUMASI METROPOLIS OF GHANA}

${ }^{1}$ Williams Ackaah*, ${ }^{2}$ Eric Aidoo. ${ }^{1}$ CSIR - Building and Road Research Institute, Kumasi, Ghana; ${ }^{2}$ Kwame Nkrumah University of Science and Technology, Kumasi, Ghana

10.1136/injuryprev-2021-safety.230

In Ghana, approximately $12 \%$ of all road traffic fatalities occur at junctions and the cause of these crashes, as assigned by the traffic police, include signal violation. Red light running place the violator and other road users at risk of road traffic crash. The main aim of the research work was to undertake baseline study to establish the current level of red light running through a direct observation survey and determine the risk factors associated with traffic light violation in the Kumasi Metropolis of Ghana. An uninterrupted road side observational survey was conducted at ten (10) signalized intersections using pro-forma checklist. A binary logit model was employed to determine the risk factors associated with traffic light violations. Overall, drivers were observed running red in $35 \%$ of all the red phases observed. From the model, factors which influence red light running include the age and gender of the driver, presence of a passenger in the vehicle, vehicle type, junction type, cycle length of the signal and queue length. There is a need for public awareness campaigns on the dangers of red light running. The education on red light violation must be accompanied by sustained enforcement of the traffic law by the traffic police to help reduce the violation of red light. Deployment of automatic red light cameras will also go a long way in ensuring enforcement at all times.

\section{P5.011 EVALUATION OF INTERVENTIONS TO PROMOTE CHILD RESTRAINT USE AMONG KINDERGARTENERS IN CHINA}

Yixiang Peng*, Shuzhen Yan, Kaiting Zhang, Liping Li. Injury Prevention Research Center of Shantou University Medical College, Shan Tou, China

10.1136/injuryprev-2021-safety.231

Objectives To evaluate the effectiveness of parent-based child restraint system (CRS) intervention so as to promote the parents' knowledge, attitude, and use of CRS.

Methods We conducted a randomized trial with cluster sampling in 8 selected kindergarteners in Shantou and Chaozhou, China (4 from each city). Parents were randomly assigned to receive 1 of the 4 conditions: education intervention, behavioral education intervention, biomechanical visualization intervention, or control.

Results Six months after the intervention, multivariate logistic regression models showed that child gender of boy, parents' 
higher education level, accept the intervention and different interventions were had a higher probability of using CRS, in which, the probability of intervention group tending to use CRS was significantly higher than the control group (biomechanical visualization $(\mathrm{OR}=2.57,95 \% \mathrm{CI}=1.72-3.85)$, education $(\mathrm{OR}=2.27,95 \% \mathrm{CI}=1.52-3.39), \quad$ behavioral education $(\mathrm{OR}=2.01,95 \% \mathrm{CI}=1.35-3.02))$, but there was no significant difference in the pairwise comparisons between the intervention groups. The ratings on child passenger safety knowledge and attitude increased significantly in both intervention groups $(\mathrm{P}<0.001)$ but remained unchanged in the control group after the intervention, the ratings are higher in biomechanical visualization intervention group than the education intervention group $(\mathrm{p}<0.05)$.

Conclusions Both parent-based child restraint system intervention helped to promote the parents' knowledge, attitude, and use of CRS. Biomechanical visualization could be an effective intervention to improve the use of CRS in the future.

\section{P5.012 A TIME-SERIES STUDY OF CHILD PASSENGER SAFETY IN SHANTOU, CHINA}

Liping Li*, Shuzhen Yan. Injury Prevention Research Center, Shantou University Medical College, Shan Tou, China

10.1136/injuryprev-2021-safety.232
Objective To explore the prevalence of child passenger safety behaviors and safety restraint use when children were riding so as to promote the use of child restraint system (CRS) in Shantou, China.

Methods Four cross-sectional studies were conducted in 2012, in 2015, in 2017 and in 2019. Hospitals, kindergartens, primary and secondary schools, highway toll stations were randomly selected as roadside observation sites. Data were collected on the use of safety restraint measures for children aged 17 and younger in the urban area of Shantou, China.

Results From 2012 to 2019, child passenger safety behaviors increased. The rate of safety restraints use increased from $9.6 \%$ in 2012 to $22.6 \%$ in 2019 ( $\mathrm{P}<0.001$ ), among which the rate of CRS use increased from $0.7 \%$ in 2012 to $8.2 \%$ in 2019 ( $\mathrm{P}<0.001)$, the rate of seatbelt use increased from $8.9 \%$ in 2012 to $14.4 \%$ in 2019 ( $\mathrm{P}<0.001$ ). The proportion of children sitting in the front row decreased from $30.3 \%$ in 2012 to $10.2 \%$ in 2019 ( $\mathrm{P}<0.001$ ). The percentage of children sitting on an adult's legs decreased from $16.7 \%$ in 2012 to $8.2 \%$ in 2019 ( $\mathrm{P}<0.001)$.

Conclusion From 2012 to 2019, the rate of CRS use and child passenger safety behaviors increased in Shantou, China. However, the CRS usage rate is still low. Corresponding measures need to be taken to ensure the safety of children when riding in the future. 